Revue de droit comparé du travail et de la sécurité sociale

2| 2018

Harcèlement moral au travail en jurisprudence

comparée

\title{
Le harcèlement au travail dans la jurisprudence japonaise
}

Kanta Owada

(2) OpenEdition

Journals

Édition électronique

URL : https://journals.openedition.org/rdctss/1945

DOI : $10.4000 /$ rdctss. 1945

ISSN : 2262-9815

Éditeur

Centre de droit comparé du travail et de la sécurité sociale

Édition imprimée

Date de publication : 1 juin 2018

Pagination : 40-49

ISSN : 2117-4350

Référence électronique

Kanta Owada, «Le harcèlement au travail dans la jurisprudence japonaise », Revue de droit comparé du travail et de la sécurité sociale [En ligne], 2 | 2018, mis en ligne le 01 novembre 2021, consulté le 13 novembre 2021. URL : http://journals.openedition.org/rdctss/1945; DOI : https://doi.org/10.4000/ rdctss. 1945

\section{(c) $\Theta \Theta \Theta$}

Revue de droit comparé du travail et de la sécurité sociale est mise à disposition selon les termes de la Licence Creative Commons Attribution - Pas d'Utilisation Commerciale - Pas de Modification 4.0 International. 


\section{LE HARCÈLEMENT AU TRAVAIL DANS LA JURISPRUDENCE JAPONAISE}

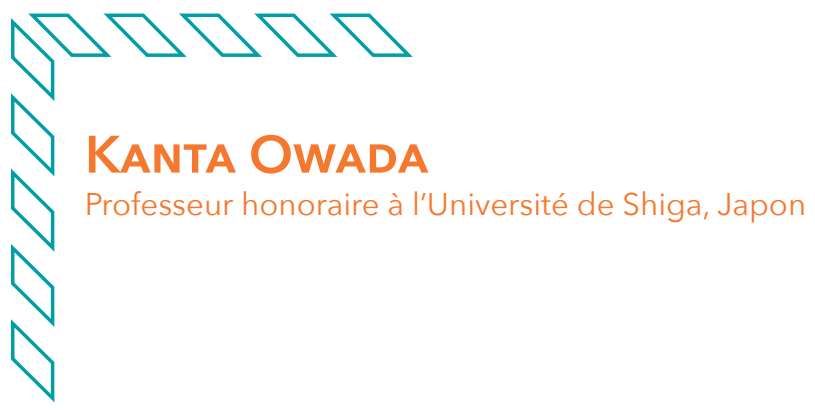

e harcèlement moral au travail est un sujet d'une actualité brûlante au Japon. En effet, selon les statistiques de I'Administration du travail japonaise, le harcèlement au travail ${ }^{1}$ est l'un des motifs de recours les plus fréquents auprès des Directions départementales du travail (70 917 cas pour l'année 2016, soit 22,8\% de l'ensemble des recours)². Par ailleurs, le nombre de décès causés par des accidents du travail dans le secteur privé était de 928 en 2016 pour 54,36 millions de salariés. Or, parmi ces victimes, 261 sont des cas de Karôshi (mort subite par surcharge de travail) et 84 se révèlent être des suicides causés par des risques psychosociaux (Karôjistasu). Le nombre des suicides a malheureusement augmenté ces dernières années. Le rapport annuel réalisé par les services de la police, en 2016, montre que le nombre total de suicides était de 21897 dont 6324 salariés $(28,9 \%)^{3}$. L'analyse des motifs de suicide montre que 1978 cas étaient liés à des problèmes de travail ou à des échecs d'ordre professionnel, aux relations humaines, aux changements dans le milieu travail et à la fatigue du travail.

1 Le harcèlement sexuel n'est pas inclus dans ces chiffres. II est traité séparément tant dans la statistique que dans le recours auprès de l'administration du travail.

2 II faut noter que le harcèlement fait partie intégrante de certains contentieux relatifs au licenciement ou à la retraite, etc.

3 Pour des salariés hommes, le taux de suicide est de 193,8 pour cent mille (48,8 pour les femmes, 128,1 pour les hommes et les femmes ensemble). Selon C. Cohidon et al. Suicide et activité professionnelle en France : premières exploitations de données disponibles, Institut de veille sanitaire, 2010, p. 4, le taux annuel de suicide en France pour des salariés hommes entre 1976 et 2002 est de 25,1 \% (pour les femmes, non calculé. 
La Commission de recherche pour la santé mentale au travail réunie en 2010 par le Ministère de la santé, du travail et de la protection sociale a représenté le processus conduisant les salariés à se suicider selon trois modèles:

(1) MutationFatigue $\rightarrow$ Relation humaine +Dépression $\rightarrow$ Suicide

(2) Promotion $\rightarrow$ Fatigue $\rightarrow$ Échec au travail $\rightarrow$ Relation humaine $\rightarrow$ Suicide

(3) Harcèlement au travail $\rightarrow$ Dépression $\rightarrow$ Suicide.

Il est également reconnu que les suicides des salariés résultent notamment de problèmes de management dans l'entreprise. Ainsi, bien que sachant que les longues heures de travail augmentent le taux de mortalité des salariés, seulement la moitié des salariés sont rémunérés pour l'intégralité des heures supplémentaires effectuées. Face à cette situation, il n'existe pas de réglementation véritablement efficace. Seules les recommandations de l'administration du travail conseillent de plafonner annuellement les heures supplémentaires à 360 heures (soit 45 heures par mois); mais en réalité, il ne s'agit pas d'une mesure ayant force obligatoire.

La politique de l'administration du travail met en revanche l'accent sur l'amélioration de la sensibilisation des salariés envers les risques psychosociaux au travail. Lorsqu'ils sont identifiés', ils ne sont traités qu'à un niveau individuel et seule une solution individuelle sera encouragée, ce qui n'est pas en phase avec des risques psychosociaux qui traduisent en réalité des risques organisationnels. Cela montre que la politique du travail japonaise a tendance à négliger le rôle des syndicats ainsi qu'une approche collective de la prévention des atteintes à la santé au travail, particulièrement les risques psychosociaux au travail. Si les syndicats sont organisés à l'intérieur de l'entreprise, et à ce titre appelés "syndicatsmaisons ", malgré un taux élevé de syndicalisation (17,1 \% en 2017), ils n'ont pas assez d'influence concernant ces nouveaux risques décrits comme des risques psychosociaux. Or, à travers ces derniers, se pose en filigrane la question de la réalité de la représentativité des syndicats auprès des salariés, notamment dans le cadre de la législation sur la santé au travail.

\section{I - LA LÉGISLATION DU TRAVAIL ET LE HARCÈLEMENT ${ }^{4}$}

\section{A - L'ABSENCE DE LÉGISLATION GÉNÉRALE SUR LE HARCÈLEMENT}

Malgré la connaissance de la gravité de la situation, il n'existe pas au Japon de réglementation spécifique sur le harcèlement. Une loi spéciale a cependant été adoptée pour s'appliquer dans certains cas. En 1997, à la suite de la modification de la loi Securing, Etc. of Equal Opportunity and Treatment between Men and Women in Employment ${ }^{5}$, des

4 Le nom de la loi est cité en anglais, tel que traduit par le site Japanese Law Translation, dépendant du ministère de la Justice, http://www.japaneselawtranslation.go.jp/

5 http://www.mhlw.go.jp/file/06-Seisakujouhou-11900000-Koyoukintoujidoukateikyoku/0000133458.pdf 
dispositions ont été adoptées pour faire face aux problèmes causés par le harcèlement sexuel au travail' notamment en consacrant l'obligation de l'employeur de prévenir cette forme de harcèlement .

À l'exception du harcèlement sexuel, en l'absence de législation spécifique au harcèlement, pour obtenir des dommages-et-intérêts devant les juridictions du travail, les victimes doivent se fonder sur des dispositions de portée générale. II en est ainsi de l'obligation de l'employeur d'assurer la sécurité et la santé des salariés ${ }^{7}$, ou alors, depuis 2007, lors de l'adoption de la loi Labor Contracts Act, l'obligation contractuelle de l'employeur d'assurer la sécurité et la santé des travailleurs ${ }^{8}$, ou encore, depuis 2017, l'interdiction des discriminations contre les femmes enceintes ou les femmes et les hommes en congé parental ${ }^{9}$. La notion de harcèlement au Japon est par ailleurs considérablement fragmentée.

\section{B - LA FRAGMENTATION DES NOTIONS}

Mise à part l'hypothèse d'un harcèlement sexuel, il n'existe pas de notion juridique relative au harcèlement en général. Dans ce contexte, en 2012, l'administration du travail a adopté une définition et édictée des lignes de conduite concernant le "Power Harassement » («Pawa-hara »). Cette position vise à prévenir en partie les harcèlements caractérisant un Power Harassement.

Le Power Harassment suppose l'existence de prérogatives dont disposent les supérieurs hiérarchiques. Lorsqu'ils en abusent, cette situation peut être qualifiée de Power Harassment. Pour se défendre et échapper à la mise en jeu de leur responsabilité, les harceleurs sont parfois tentés de prétendre, que leurs actions s'inscrivent dans le cadre de leurs missions et qu'ils ont simplement agi pour diriger leurs subordonnés.

La notion de "Power Harassement » suppose donc une relation hiérarchique entre supérieurs et subalternes. Toutefois cette condition, trop restrictive, suscite des critiques. Elle a le désavantage de rétrécir le champ d'application de la notion de harcèlement qui est, par exemple, un obstacle à la qualification de harcèlement lorsque les faits émanent de clients (harcèlement externe). Dans ce cas de figure, l'administration du travail continue néanmoins d'évoquer la notion de harcèlement.

6 Les employeurs doivent prendre les mesures nécessaires en matière de gestion du personnel pour conseiller les travailleurs en matière de harcèlement sexuel sur le lieu de travail afin qu'ils ne subissent aucun préjudice en raison de ce harcèlement sexuel (Article 11).

7 «Toute personne qui a intentionnellement ou par négligence porté atteinte à un droit d'autrui ou à un intérêt juridiquement protégé d'autrui est tenue d'indemniser tout dommage en résultant » (Article 709 du Code civil).

8 Dans le contrat de travail, l'employeur s'engage à prendre les mesures nécessaires pour permettre au salarié de travailler tout en assurant sa sécurité physique (Article 5).

9 Article 11(2) de Securing, Etc. of Equal Opportunity and Treatment between Men and Women in Employment Act Article 25 Childcare Leave, Caregiver Leave and Other Measures for the Welfare of Workers Caring for Children or Other Family Members Act. 
La notion de harcèlement au travail au Japon est fragmentée car pour en désigner les différentes formes, beaucoup de termes existent aujourd'hui. Plus de trente mots sont utilisés, quelques-uns sont à souligner:

- «Maternity Harassment» : harcèlement contre les femmes enceintes ou ayant accouché.

- «Parental Harassment » : harcèlement contre les femmes ou hommes en congé parental ou demandant à en bénéficier.

- "Karôshi (Long working)" : harcèlement en raison d'une longue durée de travail comme l'ajout de plus de 100 heures supplémentaires par mois.

- «New Employee Harassment» : harcèlement contre le travailleur novice embauché sans expérience et sans connaissance de l'activité de l'entreprise.

- «Academic Harassment » : harcèlement par l'enseignant du personnel administratif ou de l'étudiant.

- «Age Harassment» : harcèlement en raison de l'âge contre les travailleurs seniors ou les jeunes salariés.

- «Marriage Harassment » : harcèlement contre les célibataires.

- «Alcohol Harassment » : harcèlement contre ceux qui n'aiment pas boire ou ne savent pas boire.

- «Smoke Harassment» : harcèlement par les fumeurs

- «Social Media Harassment » : harcèlement via les réseaux sociaux.

- "Doctor Harassment »: harcèlement par les médecins, y compris les accidents médicaux.

- «Monster Parent » : harcèlement des instituteurs par les parents d'élèves.

Les victimes de harcèlement, qui souhaiteraient engager la responsabilité des auteurs, doivent établir l'existence des actes de harcèlement afin d'obtenir des dommages et intérêts. En effet, ils doivent établir la preuve du type de harcèlement dont ils se plaignent. Or, l'un des inconvénients majeurs de la fragmentation de la notion de harcèlement est l'absence d'une notion générale. Il est donc essentiel d'établir une notion unique de harcèlement qui pourrait s'appliquer à toutes les situations décrites.

\section{II - LA JURISPRUDENCE EN FONCTION DES CATÉGORIES DE HARCÈLEMENT}

Les actes de harcèlement au travail peuvent être classés en cinq catégories : harcèlement dans l'accomplissement des tâches quotidiennes (A), harcèlement par la direction des ressources humaines $(B)$, harcèlement par des attitudes offensantes $(C)$, harcèlement en raison de discriminations (D), harcèlement par des personnes extérieures à l'entreprise (E). Chacune de ces catégories de harcèlement fait l'objet de différentes décisions constituant la jurisprudence japonaise à ce sujet.

\section{A - HARCÈLEMENT DANS L'ACCOMPLISSEMENT DES TÂCHES QUOTIDIENNES AU TRAVAIL}

Cette catégorie harcèlement et la jurisprudence qui s'est développée regroupent les cas de Karôshi ou de suicide par surcharge de travail (Karôjisastsu). Ces situations dramatiques sont causées par de longues durées de travail (1), une charge de travail 
extrême (2), le manque de soins ou de considération du travailleur nouvellement embauché (3), des sanctions disciplinaires inappropriées (4), la position hiérarchique dans le milieu de l'enseignement (5).

\section{Karôshi et longueur excessive de la durée de travail}

La plus remarquable des décisions en matière de Karôshi est celle relative à l'affaire Dentsu du 24 mars $2000^{10}$. Dans cet arrêt de la Cour suprême, un jeune salarié avait été contraint de travailler 147 heures supplémentaires par mois. L'existence d'un harcèlement a été reconnue derrière la durée du travail à laquelle était soumis ce salarié qui s'est finalement suicidé. En l'espèce, le jeune Ichiro Oshima, a commencé à travailler le $1^{\text {er }}$ avril 1990 au sein de l'entreprise Dentsu après l'obtention de son diplôme universitaire. II était en charge de la planification commerciale et des événements pour les promotions radio gérées par Dentsu. S'il pouvait retourner à son domicile dans la journée, en réalité, il n'y revenait que vers une ou deux heures du matin. En août1990, le nombre de soirées passées au travail a augmenté. Dès le mois de novembre, il lui arrivait parfois de ne plus rentrer chez lui. En juillet 1991, le chef de l'équipe du jeune homme a effectivement estimé qu'il ne travaillait pas forcément dans de bonnes conditions de travail et ne les vivaient pas bien. Il a d'ailleurs commencé à s'exprimer envers son équipe en disant par exemple: «Parfois, je n'arrive pas à reconnaître ce que je fais et ce que je dois faire » ou encore « je ne peux pas dormir, je me réveille après seulement deux heures de sommeil. Mon insomnie a empiré depuis août et je n'en connais pas la raison ». Le jeune homme s'est alors pendu, juste après avoir achevé un travail sur un événement pour une émission de radio dont il était responsable.

Son père et sa mère ont déposé plainte contre Dentsu en alléguant que le suicide de leur fils avait été commis en raison d'une dépression causée par l'épuisement découlant de l'extraordinaire charge de travail supplémentaire à laquelle il devait faire face. Leur action portait sur la responsabilité de l'entreprise Dentsu pour non-exécution de son obligation de veiller à la sécurité de ses salariés en vertu de l'article 415 du Code civil et pour conduite délictueuse au titre de l'article 709 du Code civil. L'arrêt rapportait aussi que la victime, qui n'aimait pas les boissons alcoolisées, subissait l'humiliation de boire de la bière versée dans une chaussure par ses supérieurs. La Cour suprême a estimé qu'une entreprise a l'obligation d'organiser le travail de telle manière qu'il n'en résulte pas de maladie mentale et physique découlant de l'accumulation de fatigue ou de contraintes excessives. Le 24 mars 2000, la Cour suprême a accordé des dommages et intérêts d'un montant de 1377050 € (168 millions V) sur le fondement de la responsabilité de l'employeur prévue par le Code civil. (Une personne qui emploie d'autres personnes sera responsable des dommages causés à un tiers par ses salariés à l'occasion de l'exécution de cette activité (Article 715 du Code civil).

En 2016, dans la même société Dentsu, le jour de Noël, une jeune salariée s'est suicidée en raison d'un Karôshi. Elle avait travaillé environ 105 heures supplémentaires un mois avant sa mort. L'entreprise avait faussé la déclaration des heures supplémentaires en enregistrant une durée du travail de 70 heures qui était la limite conventionnelle des

10 Cour Suprême 24 mars 2000, RH (Rodo Hanrei, Revue des arrêts du droit du travail), 779. Dentsu est l'une des plus grandes entreprises de publicité mondialement connue. Voir L. Lerouge, Risques psychosociaux et système japonais de prévention des risques professionnels, Documents de travail, COMPTRASEC, 2013. https://halshs.archives-ouvertes.fr/halshs-00818332/document 
heures supplémentaires mensuelles en vertu de la convention collective négociée par les partenaires sociaux de l'entreprise. Dans cette affaire, le supérieur hiérarchique de la salariée lui avait reproché 20 heures de travail jugées inutiles pour la société et l'avait réprimandé sur son apparence physique car elle ne devait pas se rendre au travail en ayant les yeux congestionnés. Le 5 octobre 2017, le Tribunal du district de Tokyo a condamné la société Dentsu au paiement d'une amende de $4100 €(\backslash 500000)$ pour infraction à la gestion de la durée du travail prévue dans la loi Labor Standards Act.

Ainsi, Karôshi et harcèlement se croisent. Le harcèlement précipite le Karôshi et, dans le même temps, les conditions menant au Karôshi deviennent une situation de harcèlement. L'employeur est responsable des conditions de travail. Plus particulièrement au Japon, il est responsable des effets du temps de travail sur la santé.

Dans une autre affaire, un jeune chauffeur s'est suicidé à la suite de longues heures de travail supplémentaires. Dans son entreprise de transport, l'accord collectif prévoyait un nombre maximal de 70 heures supplémentaires mensuelles qui pouvaient être étendues à 100 heures dans des cas exceptionnels. En pratique, le chauffeur avait effectué 63 heures et 45 minutes en heures supplémentaires en avril 2009, 110 heures et 15 minutes en mai, 99 heures et 50 minutes en juin, 129 heures et 50 minutes en juillet, 103 heures et 55 minutes en août et 102 heures 30 minutes en septembre. II a travaillé de nuit à plusieurs reprises chaque mois (après 22 heures; respectivement 2, 2, 1, 16, 9, 6 fois). Le supérieur hiérarchique lui avait reproché d'avoir commis quelques petites erreurs que les nouveaux salariés ont tendance à faire. À la suite de cela, il est tombé en dépression et s'est suicidé. Le Tribunal du district de Sendai condamna la société à payer une indemnité pour violation de l'obligation de protéger la santé et la sécurité de l'employé11.

\section{2 - Surcharge de travail et harcèlement}

Une société de courtage vendait des obligations au porteur et répartissait les sommes à atteindre entre chaque salarié. Ces sommes leur semblaient très difficiles à atteindre. Lorsqu'un salarié était dans l'impossibilité de réaliser l'objectif fixé, son supérieur lui demandait de vendre son propre véhicule pour assurer des chiffres de ventes. La santé mentale de l'un des salariés en a été gravement impactée, le conduisant à commettre un suicide. Le Tribunal du district de Tokyo a considéré que les sommes à atteindre étaient trop importantes et que les paroles du supérieur à son encontre avaient été la cause du suicide. En conséquence, il avait ordonné à la société de verser des dommages et intérêts ${ }^{12}$.

\section{3 - Manque de soin ou de considération et effet sur la charge de travail d'un nouvel embauché}

L'un des harcèlements les plus fréquents est celui que subit le jeune nouvellement embauché. Lorsqu'il intègre le personnel de l'entreprise, il n'a ni la connaissance ni l'expérience nécessaire à son poste de travail. Le supérieur lui demande un travail, mais le jeune employé ne saura pas l'effectuer en l'état de ses connaissances. Son travail devient alors lourd et synonyme d'un poids psychologique. Ce n'est pas rare que cette surcharge de travail accule le salarié à commettre un suicide. L'employeur et les supérieurs doivent

11 Trib. Sendai, 25 juin 2013, RH, 1079.

12 Trib. Tokyo, 13 décembre 2013 non publié. 
prendre en considération la santé mentale du jeune salarié et lui attribuer des objectifs et une charge de travail, adaptés lorsqu'il débute son travail.

\section{4 - Sanctions disciplinaires et harcèlement}

Le chauffeur d'autobus d'une entreprise de transport routier avait endommagé la carrosserie de son bus à la suite d'un accrochage avec un autre véhicule. Son employeur lui a ordonné de cesser de conduire et l'a affecté au désherbage du site de dépôt d'autobus au motif qu'il fallait corriger ses erreurs de conduite. L'exécution de ce nouveau travail nécessitait une exposition durant de longues heures au soleil brûlant et au regard de ces collègues et des piétons. Le Tribunal du district de Yokohama a estimé que cette sanction disciplinaire ne pouvait pas avoir pour effet l'amélioration des techniques de conduire et a condamné la société au paiement de dommages-intérêts ${ }^{13}$.

Dans une autre entreprise, Japan Railway (JR), un conducteur a reçu l'ordre de copier dans une pièce isolée les règlements intérieurs de l'entreprise, sans moyens de communication avec son service. Cette sanction disciplinaire fut jugée constitutive d'un harcèlement par le Tribunal du district d'Osaka ${ }^{14}$.

Outre le travail imposé à titre de sanction disciplinaire, on peut citer le travail inutile. Par exemple, un supérieur a ordonné à son subordonné de traduire un livre en anglais. Ce salarié n'était pas traducteur et n'avait donc aucune expérience en matière de traduction. Il finit ce travail en deux semaines, sans que cela ne soit d'une quelconque utilité pour l'entreprise. Le Tribunal du district de Tokyo a qualifié de harcèlement cette situation de travail imposée au salarié ${ }^{15}$.

\section{5 - Harcèlement au travail en raison de la position hiérarchique dans l'enseignement ou Aca Hara (Academic Harassment)}

Les victimes de cette catégorie harcèlement sont des étudiants, des assistants ou professeurs adjoints que le professeur titulaire soumet à des évaluations, à des examens, ou à des mesures discriminatoires concernant les subventions et les promotions. II est difficile pour ces victimes de résister au professeur qui détient le pouvoir. Dans une affaire dans I'Université de médecine, un professeur avait injustement refusé de délivrer à son assistante un document nécessaire à sa fonction et avait bloqué son bureau en déposant un récipient d'eaux polluées devant la porte pour l'empêcher de faire des recherches. L'assistante, victime de ces traitements injustes, a obtenu réparation pour les actes de son professeur référent qui ont été reconnus comme constitutifs d'un harcèlement. Le tribunal lui a donné gain de cause et a reconnu la responsabilité de l'Université en raison de l'obligation d'assurer la sécurité et la santé des fonctionnaires dans l'exercice de leur métier ${ }^{16}$.

13 Trib. Yokohama, 21 septembre 1999, RH, 771.

14 Trib. Osaka, 19 septembre 2007, RH, 959.

15 Trib. Tokyo, 13 mars 2001, RH, 805.

16 Trib. 11 octobre 2000, RH, 799. 


\section{B - HARCÈLEMENT PAR LA DIRECTION DES RESSOURCES HUMAINES}

Dans ce type de situation est visé la politique managériale et le comportement de la direction susceptibles de constituer des actes de harcèlement. Ainsi, dans une compagnie d'électricité, l'employeur tenta d'écarter quelques salariés qui l'avaient critiqué. La direction se mit à contrôler les communications entre les salariés, à écouter clandestinement des conversations téléphoniques et à inspecter secrètement les vestiaires. La direction fit pression pour dissoudre les amitiés nées entre les salariés. La Cour suprême condamna cette série de mesures en les jugeant attentatoires à la liberté d'instaurer librement des relations humaines ${ }^{17}$. La politique et l'action de la direction des ressources humaines doivent respecter cette liberté entre salariés. Dans le cas inverse, il s'agit d'un harcèlement des ressources humaines.

Un autre exemple de harcèlement managérial peut résulter du remaniement $d u$ personnel. Au Japon, en général, l'employeur a tendance à trop s'appuyer sur son pouvoir de modification du contrat de travail en passant outre le consentement des salariés. En effet, il n'est guère aisé pour salarié de s'opposer au changement de lieu de travail ou de fonction qui lui est proposé. L'employeur peut ainsi abuser de ce pouvoir, au point de peser lourdement sur la vie aussi bien professionnelle que personnelle du salarié. Dans une affaire, un salarié s'était opposé à la mutation dans un autre service et officieusement proposée par son employeur. Devant ce refus, il décida de supprimer artificiellement le département dont il dépendait, et l'a déplacé au nouveau poste. Le Tribunal du district de Tokyo estima que ce changement avait occasionné beaucoup d'inconvénients à l'égard du salarié, était injuste et abusif et a annulé cette mesure ${ }^{18}$.

\section{C - HARCÈLEMENT PAR DES ATtitudes OfFEnSANTES}

En général, ce type de harcèlement consiste en des attitudes offensantes sur un plan physique ou psychologique comme par exemple des violences, des menaces, des négligences. II s'agit aussi d'obliger le travailleur à utiliser ses ressources propres pour l'exécution de la prestation de travail, de lui faire subir des plaisanteries, des calomnies et de la diffamation. Dans une telle situation, il est possible de reconnaître un cumul de responsabilités : pénale pour l'auteur des faits, et civile pour l'employeur qui devra assumer les conséquences d'un harcèlement dans son entreprise. Au Japon, en l'absence de disposition spécifique sur le harcèlement moral au travail, le droit pénal général s'applique.

Ainsi, à titre d'illustration, dans un institut de chimie, un salarié voulait se venger d'une femme employée dans le même établissement pour des motifs privés et d'ordre sentimentaux. Il enduisit l'intérieur de ses chaussures de poison. En se chaussant, elle subit de graves blessures aux pieds et aux jambes. Il fut poursuivi pour coups et blessures et fut condamné à une peine de prison de sept ans. Dans cette affaire ${ }^{19}$, ce fut la juridiction pénale qui fut compétente pour sanctionner ce comportement. Pour éviter toute mise en cause dans une situation de harcèlement, l'employeur doit prévenir ce genre de comportement en agissant en amont.

17 Cour Suprême, 5 septembre 1995, RH, 680.

18 Trib. Tokyo, 26 juin 2006, RH, 934.

19 Trib. Shizuoka, section de Numazu, 20 septembre 2013, LEX/DB 25501757. 
Plus récemment, il est reconnu que I'utilisation d'Internet peut humilier un salarié au moyen de courriels, de blogs, de chat et autres instruments numériques qui peuvent être utilisés anonymement. II n'existe pas encore au Japon de véritables développements visant à réfléchir à des méthodes efficaces pour prévenir ce type de harcèlement.

\section{D - HARCÈLEMENT EN RAISON DE DISCRIMINATIONS}

Le harcèlement discriminatoire est celui qui est constitué par des discriminations en raison du sexe, de l'âge, du handicap ou de la nationalité. II sera ici question d'envisager des cas de discriminations en raison du sexe. Le cas typique de ce qui est appelé au Japon "harcèlement sexuel » porte sur la discrimination entre les femmes et les hommes dans l'avancement hiérarchique et les promotions professionnelles.

Ainsi, dans un établissement financier, les hommes bénéficiaient d'une nomination au poste de chef de service entre 13 et 15 ans après avoir été embauchés comme agents au guichet. Ils se voyaient par la suite promus six ans plus tard au poste de chef de bureau. En revanche, à âge égal, les femmes embauchées en même temps ne pouvaient que rarement profiter d'un avancement. Une salariée avait néanmoins été nommée chef de service après 36 ans d'ancienneté et aucune représentante du sexe féminin n'était devenue chef de bureau. Pour ces raisons, les salariées de cette entreprise réclamèrent un rappel de salaire en compensation de l'absence de promotion dont elles auraient dû bénéficier compte tenu de leur ancienneté. Elles sollicitèrent également leur nomination aux mêmes postes que ceux occupés par des hommes embauchés en même temps qu'elles.

En première instance, le Tribunal du district de Tokyo fit droit à la demande de rappel de salaire, mais refusa l'avancement hiérarchique au motif que seul l'employeur a le pouvoir de décider des promotions professionnelles de ses salariés ${ }^{20}$. En appel, le Tribunal a accueilli les prétentions des requérantes pour l'intégralité de leurs demandes en considérant qu'il s'agissait du moyen le plus adéquat pour remédier à cette différence de traitement injustifiée ${ }^{21}$.

Plus récemment, des discriminations de genre ont été constatées à l'occasion du changement de nom après un mariage civil ${ }^{22}$. Ainsi, $96 \%$ de ceux qui ont changé de nom après le mariage sont des femmes ${ }^{23}$. Cela présente un lien avec la discrimination sexuelle et de harcèlement contre les femmes. En effet, si certains membres d'une assemblée délibérante ou artistes changent, en droit, leur nom après le mariage, ces personnes continuent dans la pratique à utiliser leur nom de jeune fille ou de jeune homme. En revanche, dans une entreprise, ceux qui changent leur nom après le mariage sont tenus de signifier ce changement à la direction et d'employer leur nouveau nom. Or, dans le domaine universitaire, cela désavantage considérablement les chercheurs ou les enseignants, puisque ces personnes doivent publier leurs articles en utilisant dorénavant le nom de leur conjoint. Le travail publié sous le nom de jeune fille n'est cependant pas affecté par ce changement.

20 Trib. Tokyo, 27 novembre 1996, RH, 740.

21 Tokyo, 22 décembre 2000, RH, 796.

22 'Un mari et une femme adoptent le nom de famille du mari ou de la femme conformément à ce qui est décidé au moment du mariage.'(Article 750).

23 Ministère du travail, 2016. 
La Cour suprême a admis le 16 décembre 2015 la constitutionnalité de cette règle relative au changement du nom après le mariage ${ }^{24}$. Toutefois une part croissante de la population féminine sollicite une modification de cette règle afin que chaque époux puisse conserver l'usage de son nom.

Parmi les mesures discriminatoires pouvant affecter les représentants d'un sexe, le harcèlement est très fréquent. II existe une règlementation spéciale relative au harcèlement sexuel permettant aux victimes de demander réparation. Cela témoigne de l'importance que revêt la loi dans la lutte contre le harcèlement pour le prévenir et aider les victimes. On doit prendre en considération cette leçon.

L'un des problèmes essentiels en matière de harcèlement au travail est l'absence de définition juridique générale du harcèlement moral qu'il convient de résoudre le plus rapidement possible. Le droit du harcèlement au Japon est très lié au droit de la discrimination comme dans un certain nombre de pays de langue anglaise (États-Unis, Royaume-Uni, Australie, etc.). II serait néanmoins opportun d'adopter des textes portant sur chaque type de harcèlements de manière à être plus en phase avec leur réalité et leurs caractéristiques. C'est notre mission la plus importante.

24 Cass., 16 décembre 2015, MinSyu (Revue des arrêts du droit civil), 69-8. 